\title{
Equilibrium Analysis of Dynamic Models of Imperfect Competition *
}

\author{
Juan F. Escobar \\ University of Chile ${ }^{\dagger}$
}

October 26, 2011

\begin{abstract}
Motivated by recent developments in applied dynamic analysis, this paper presents new sufficient conditions for the existence of Markov perfect equilibrium in dynamic stochastic games. The main results imply the existence of Markov perfect equilibrium provided the sets of actions are compact, the set of states is countable, the period payoff functions are upper semi continuous on the action profiles and lower semi continuous on actions taken by rival firms, and the transition function depends continuously on the actions. Additionally, if for each firm a static best reply set is convex, then the equilibrium can be taken to be in pure strategies. Sufficient conditions for the convexity of the best replies are presented and discussed. In particular, we introduce new sufficient conditions ensuring the dynamic programming problem each firm faces has a convex solution set and deduce the existence of Markov perfect equilibrium for this class of games. Our results expand and unify the available modeling alternatives and apply to several models of interest in industrial organization, including models of industry dynamics. JEL classification numbers: C73, C61, C62.
\end{abstract}

Keywords: Industry dynamics, dynamic stochastic games, Markov perfect equilibrium

\footnotetext{
*I thank Uli Doraszelski, Jon Levin, Bob Wilson, and three anonymous referees for useful comments and suggestions. This paper supersedes my previous working paper "Existence of pure and behavior strategy stationary Markov equilibrium in dynamic stochastic games" (Stanford, 2008).

${ }^{\dagger}$ Center for Applied Economics, Department of Industrial Engineering, University of Chile, Republica 701, Santiago, Chile. E-mail: jescobar@dii.uchile.cl
} 


\section{Introduction}

This paper considers infinite horizon games where at each period, after observing a payoff relevant state variable, players choose actions simultaneously. The state of the game evolves stochastically parameterized by past history in a stationary Markov fashion. The setting includes a broad class of models, including Ericson and Pakes's (1995) model, as well as more general dynamic models of imperfect competition.

We present our main existence results in an abstract way and offer several applications in Section 4. A strict implication from our main result is the following. A dynamic stochastic game possesses a behavior strategy Markov perfect equilibrium if the sets of actions are compact, the set of states is countable, the period payoff functions are u.s.c.-l.s.c. in current actions (or, more generally, depend on actions in a sense similar to that considered by Dasgupta and Maskin 1986), and the probability distribution of the next state depends continuously on the actions chosen. Moreover, if for each player a static best reply set is convex, then the equilibrium can be additionally shown to be in pure strategies.

As in received work (Horst 2005, Doraszelski and Satterthwaite 2010), in order to obtain existence in pure strategies we impose convexity restrictions on the dynamic game. Our result requires the game to have convex best replies, meaning that for all rivals' actions and all (bounded) continuation functions, each firm's static best reply set is convex. This condition resembles (and indeed reduces to) the standard convexity restriction imposed on the payoff functions in strategic form games to ensure the existence of Nash equilibrium. We state independent, sufficient conditions ensuring the convexity of the best replies. Our first sufficient condition is the uniqueness of the set of best replies, a condition requiring best reply sets to be single valued. This condition reduces to the convexity condition introduced by Doraszelski and Satterthwaite (2010) in an industry dynamics model. The second sufficient condition, satisfied by the so called games with concave reduced payoffs, ensures that each player's maximization problem is concave and so best replies are convex valued. While these two conditions do not cover all the games having convex best replies, they significantly broaden the modeling alternatives offered by existent results.

There are several applications of our main results; Section 4 provides a few. We analyze an industry dynamics model similar to that introduced by Ericson and Pakes (1995). Doraszelski and Satterthwaite (2010) have recently studied a version of the Ericson-Pakes model and introduced a condition, named the unique investment choice condition (UIC), to guarantee equilibrium existence. Under Doraszelski and Satterthwaite's (2010) UIC condition, best replies are single valued and thus our convexity restrictions are met. Moreover, we provide a 
new alternative condition for existence in the Ericson-Pakes model and discuss how this new condition permits modeling alternatives uncovered by Doraszelski and Satterthwaite's (2010) analysis. In particular, in contrast to Doraszelski and Satterthwaite's (2010) framework, our results allow for multidimensional investment decisions and complementarities among firms' investments.

We also study a Markov Cournot game, in which firms compete in quantities and at each round a decision-controlled demand shock is realized. We provide sufficient conditions ensuring equilibrium existence. We show how restrictions on how payoffs are affected by rivals' actions and on how the transition depends on action profiles make existent results unsatisfactory (Horst 2005, Amir 1996, Nowak 2007). Notably, in order to ensure equilibrium existence, we do not need to restrict the number of game players nor do we need to assume the transition function is linear in action profiles. We also consider a version of the Markov Cournot game in which firms have fixed costs and show results ensuring existence of behavior strategy equilibrium.

Finally, our results are also applied to an incomplete information dynamic model extensively studied recently (e.g. Bajari, Benkard, and Levin 2007, Doraszelski and Escobar 2010).

Dating back to Shapley (1953), several authors have studied the problem of equilibrium existence in dynamic stochastic games. Until recently, most of the researchers provided conditions for the existence of mixed or correlated equilibria. Among these, Mertens and Parthasarathy (1987), Nowak and Raghavan (1992), and Duffie, Geanakoplos, Mas-Colell, and McLennan (1994) constitute important contributions which neither generalize nor are generalized by our results.

Two strands of the literature are more closely related to this work. First, Horst (2005), Doraszelski and Satterthwaite (2010), and Nowak (2007) deal with the pure strategy equilibrium existence problem. Some of these results cover state spaces uncovered by our results. Although our main result is formally unrelated to these authors', this paper identifies convexity conditions that significantly expand and unify the modeling alternatives available. Indeed, a game satisfying any of the convexity conditions imposed by those authors has convex best replies as required by our main result. Moreover, games as considered by Horst (2005) and Nowak (2007) are games with concave reduced payoffs and so, according to Lemma 2, have convex best replies 11 This work contributes to this literature by identifying convexity restrictions that significantly generalize the conditions so far available.2

\footnotetext{
${ }^{1}$ Bernheim and Ray (1989) and Dutta and Sundaram (1992) derive pure strategy results formally unrelated to ours. For a class of dynamic models, they restrict the strategy sets so that best replies are single valued and the games therefore satisfy the convexity restrictions required by our analysis.

${ }^{2}$ While Amir (1996) and Curtat (1996) restrict their attention to supermodular stochastic games, they do
} 
These results also contribute to the literature on dynamic games with countable state spaces. Federgruen (1978) and Whitt (1980) provide existence results which are corollaries to our main behavior strategy result, Theorem 6, in that they do not permit payoffs to be discontinuous. In particular, they do not deal with the problem of pure strategy existence, nor do they answer whether a convexity condition as the one here imposed could be satisfied by a nontrivial class of models.

The paper is organized as follows. Section 2 presents the model. Section 3 presents and discusses the main theorem. Section 4 provides a number of applications of our results. Section 5 concludes. Additional material has been relegated to the Appendix.

\section{Set Up}

In this section we introduce our dynamic game model and define our equilibrium notion. As frequently studied in Industrial Organization, we consider a dynamic stochastic game played by a finite set of firms. In each round of play, there is a payoff relevant state variable (e.g. the identity of the incumbent firms). The state variable evolves stochastically, and firms can influence its evolution through actions (e.g. by entering or exiting the market). The goal of each firm is to maximize the expected present value of its stream of payoffs.

\section{$2.1 \quad$ Model}

There is a finite set of firms denoted by $I$. At the outset of period $t=1$, firms are informed about the initial state of the game, $s_{1}$. Then they pick their actions $a_{1}=\left(a_{1}^{i}\right)_{i \in I}$ simultaneously. At the outset of period $t=2$, firms are informed of the new state of the game $s_{2}$ and then pick simultaneously their actions $a_{2}=\left(a_{2}^{i}\right)_{i \in I}$. And so on for $t \geq 3$.

The state space is $S$ and we assume that $S$ a countable set. For each firm $i$, the set of actions $A_{i}$ is compact and contained in a linear metric space. In most applications, we will assume that $A_{i}$ is contained in $\mathbb{R}^{L^{i}}$, where $L^{i}$ is a natural number, but it will be useful to allow some more generality when studying models of imperfect competition in which firms receive private technology shocks (see Section 4.3).

When firms make decisions at round $t$, they know the sequence of past states $s^{1}, \ldots, s^{t}$, and their action profile $a^{t}$ determines the distribution of the state in the next round $s^{t+1}$.

need to impose convexity conditions that, as explained in Subsection 3.2. cannot be deemed as less stringent than mine. 
Moreover, the transition function takes the form

$$
\mathbb{P}\left[s_{t+1} \in B \mid a_{t}, \ldots, a_{1}, s_{t}, \ldots, s_{1}\right]=Q\left(B ; a_{t}, s_{t}\right)
$$

where $B \subseteq S$. We will abuse notation writing $Q\left(s^{\prime} ; a_{t}, s_{t}\right)=\mathbb{P}\left[s_{t+1}=s^{\prime} \mid a_{t}, \ldots, a_{1}, s_{t}, \ldots, s_{1}\right]$. Given realized sequences of actions $\left(a_{t}\right)_{t \geq 1}$ and states $\left(s_{t}\right)_{t \geq 1}$, the total payoff for firm $i$ is the discounted sum of period payoffs

$$
\sum_{t=1}^{\infty}\left(\delta^{i}\right)^{t-1} \pi^{i}\left(a_{t}, s_{t}\right)
$$

where $\delta^{i} \in\left[0,1\left[\right.\right.$ is the discount factor, and $\pi^{i}(a, s)$ is the per period payoff function. We assume that $\pi^{i}$ is bounded for all $i$. Let $\pi_{l}^{i}$ and $\pi_{u}^{i}$ be, respectively, the lower and upper bounds for the function $\pi^{i}$ and denote $\left\|\pi^{i}\right\|_{\infty}=\sup _{a \in A, s \in S}\left|\pi^{i}(a, s)\right|$. This completes the description of our dynamic stochastic game.

This dynamic stochastic game model is very flexible and, indeed, several models widely used in the literature fit into this framework (Maskin and Tirole 1988, Ericson and Pakes 1995). Applications and examples will be discussed in Section 4.

\subsection{Markov Perfect Equilibria}

We will now present the equilibrium notion we will work with. One may study subgame perfect equilibria of our dynamic model, but recent research has focused on Markov perfect equilibria. Markov perfect equilibria is a class of subgame perfect equilibrium strategies in which players condition their play only on payoff relevant information 3 The idea is that, in a given round, firms choose actions depending on the current state with the purpose of maximizing the sum of current and future expected discounted payoffs.

A Markov strategy for firm $i$ is a function $\bar{a}^{i}: S \rightarrow A^{i}$ mapping current states to actions. Thus, a Markov strategy defines a dynamic game strategy in which in each round $t$ firm $i$ chooses action $\bar{a}^{i}\left(s^{t}\right)$, where $s^{t}$ is the shock realized in round $t$. A tuple of Markov strategies $\left(\bar{a}^{i}\right)_{i \in I}$ is a Markov perfect equilibrium if it is a subgame perfect equilibrium of the dynamic game. In a Markov perfect equilibrium, while firms condition their play only on the current state, they may deviate to arbitrary strategies conditioning on the whole transpired history. We will also consider behavior Markov perfect equilibria, defined as a subgame perfect equi-

\footnotetext{
${ }^{3}$ Several arguments in favor of this restriction can be given, and Maskin and Tirole (2001) provide and insightful discussion.
} 
libria in which each firm $i$ uses a strategy $\bar{a}^{i}: S \rightarrow \Delta\left(A^{i}\right)$ that maps current states to a distribution over actions.

\section{The Main Result}

In this section we present our main existence result, Theorem 1. After stating it, a brief discussion is presented and its proof is provided.

\section{$3.1 \quad$ Statement}

As in many dynamic models, dynamic programming tools will be useful to analyze our set up. We thus define

$$
\Pi^{i}\left(a, s ; v^{i}\right)=\pi^{i}(a, s)+\delta^{i} \sum_{s^{\prime} \in S} v^{i}\left(s^{\prime}\right) Q\left(s^{\prime} ; a, s\right)
$$

where $a \in A, s \in S$, and $v^{i}: S \rightarrow \mathbb{R}$ is a bounded function. The number $\Pi^{i}\left(a, s ; v^{i}\right)$ is the total expected payoff for player $i$, given that the current state is $s \in S$, the current action profile is $a \in A$, and the continuation payoff, as a function of the next state $s^{\prime} \in S$, is $v^{i}\left(s^{\prime}\right)$. Intuitively, fixing a state $s$ and continuation value functions $\left(v^{i}\right)_{i \in I}$, the functions $\Pi^{i}\left(\cdot, s ; v^{i}\right), i \in I$, define a static game in which firms' action profiles are $a \in A$. As we will see in Section 3.3, the Markov perfect equilibrium requirement, on the one hand, restricts continuation value functions and, on the other hand, induces Nash equilibrium behavior in the corresponding family of static games.

To guarantee the existence of Markov perfect equilibrium, we will impose convexity and regularity restrictions on our dynamic game. The dynamic stochastic game is said to have convex best replies if for all $i$, all $s \in S$, all $a^{-i}$ in $A^{-i}$, and all bounded function $v^{i}: S \rightarrow$ $\left[\frac{\pi_{l}^{i}}{1-\delta^{i}}, \frac{\pi_{u}^{i}}{1-\delta^{i}}\right]$ the best reply set

$$
\arg \max _{x^{i} \in A^{i}} \Pi^{i}\left(\left(x^{i}, a^{-i}\right), s ; v^{i}\right)
$$

is convex. This condition says that the static optimization problem in which each firm $i$ chooses an action $x^{i} \in A_{i}$ with the purpose of maximizing its total payoffs has a convex solution set, given the profile played by its rivals $a_{-i}$, the current state $s$ and the continuation value function $v^{i}$ 
It will also be useful to impose some continuity restrictions on the payoffs and the transition. The game is transition continuous if the transition function $Q$ is setwise continuous in $a \in A$ : for every $B \subseteq S$ and $s \in S, Q(B ; a, s)$ is continuous in $a \in A$ (Royden 1968, Chapter 11.4). The game is upper semi continuous if for all $i$, the function $\pi^{i}(a, s)$ is upper semi continuous in $a$ : for all $i$, all $s$, and all sequence $a_{n} \rightarrow a$ in $A$

$$
\limsup _{n \rightarrow \infty} \pi_{n}^{i}\left(a_{n}, s\right) \leq \pi^{i}(a, s)
$$

Finally, we say that the game is weak lower semi continuous if for all $i$, all $s \in S$, and all sequence $a_{n}^{-i} \rightarrow a^{-i}$ in $A^{-i}$, there exists a sequence $a_{n}^{i} \rightarrow a^{i}$ in $A^{i}$

$$
\liminf _{n \rightarrow \infty} \pi^{i}\left(a_{n}, s\right) \geq \pi^{i}(a, s)
$$

We also say that $\pi^{i}(a, s)$ is weak lower semi continuous if the condition above holds $4^{4}$ Observe that if $\pi^{i}(a, s)$ is lower semi continuous in $a^{-i}$ then it is weak lower semi continuous $5^{5}$ The basic idea behind the definition of weak lower semi continuity is that if firm $i$ attains a period payoff $\pi^{i}(a, s)$ given actions $a$ and rivals deviate slightly from $a^{-i}$ to $a_{n}^{-i}$, then firm $i$ can attain virtually the same payoff by playing an action $a_{n}^{i}$ close to $a^{i}$.

The following is our main existence result.

Theorem 1 If the dynamic stochastic game is transition continuous, upper semi continuous, and weak lower semi continuous, then it possesses a Markov perfect equilibrium provided it has convex best replies.

A number of comments on the theorem and its assumptions can be made; a complete discussion is presented in the following subsection.

\subsection{Discussion and Sufficient Conditions}

The convexity assumption is a restriction on a static best reply set. If we rely on Kakutani's fixed point theorem to obtain the existence of a profile satisfying (at least) a static Nash equilibrium condition, this kind of convexity restriction is virtually unavoidable. Indeed, in

\footnotetext{
${ }^{4}$ Upper semi continuity and weak lower semi continuity allow us to ensure best replies are closed. Similar conditions appear in Dasgupta and Maskin (1986); in particular, weak lower semi continuity implies their graph continuity restriction. Related notions are studied in the math literature (Rockafellar and Roger 2004, Chapter 7).

${ }^{5}$ Note that if $\pi^{i}(a, s)$ is lower semicontinuous in $a^{-i}$ and upper semi continuous in $a$, then it is a continuous function of $a^{-i}$.
} 
strategic form games, to ensure pure strategy existence, the convexity of the best reply set is imposed for every strategy followed by rivals. In our dynamic setting, that restriction is imposed not only for every strategy followed by rivals, but also for every continuation value function 6

The game will have convex best replies whenever the set of maximizers 3.1 is a singleton; in this case we say the game has single valued best replies. Games studied by Horst (2005) and Doraszelski and Satterthwaite (2010), among others, have single valued best replies. Section 4 presents models extensively employed in the applied IO literature where this kind of uniqueness restriction can be exploited.

Before presenting a sufficient condition for the convexity of the best replies, we will provide some more structure to the main setting. The set of actions of each player $i, A_{i}$, will be a convex set contained in $\mathbb{R}^{L^{i}}$, where $L^{i}$ is a natural number. We will also assume that $\pi^{i}(a, s)$ and $Q\left(s^{\prime} ; a, s\right)$ are twice continuously differentiable respect to $a^{i} \in A_{i}$; denote the Hessian matrices with respect to $a^{i} \in A^{i}$ by $\pi_{i i}^{i}(a, s)$ and $Q_{i i}\left(s^{\prime} ; a, s\right)$ respectively.

For a given real valued symmetric square matrix $M$, we consider the real number

$$
\operatorname{mev}(M)=\max \{\lambda \mid \lambda \text { is an eigenvalue of } M\}
$$

To see the relevance of the maximum eigenvalue of a symmetric matrix in our context, recall that for a given column vector $x$,

$$
x^{\prime} M x \leq \operatorname{mev}(M)\|x\|_{2}^{2},
$$

where $\|\cdot\|_{2}$ is the Euclidean norm (Section 9.4 in Aleskerov, Ersel, and Piontkovski 2011). We can therefore interpret the maximum eigenvalue of a symmetric matrix $M, \mathbf{m e v}(M)$, as a measure of how negative definite the matrix $M$ is. In our dynamic model, it will be useful to impose the concavity (with respect to own actions ) of the sum of current and continuous payoffs, and measuring concavity through the maximum eigenvalue of each term will prove useful.

More concretely, we say that the game has concave reduced payoffs if for all $i$, the function

\footnotetext{
${ }^{6}$ Unless one gives some more structure to the game, little can be said about continuation functions which are solutions to a dynamic programming condition. This resembles the analysis of static games: unless one adds some more structure to a (say Bayesian) game, there is no way to impose the convexity restriction on a smaller set of rivals' strategies.
} 
$\pi^{i}(a, s)$ is concave in $a^{i}$ and for all $(a, s) \in A \times S$ either

$$
\left(\pi_{u}^{i}-\pi_{l}^{i}\right) \sum_{s^{\prime} \in S} \max \left\{0, \operatorname{mev}\left(Q_{i i}\left(s^{\prime} ; a, s\right)\right)\right\}-\operatorname{mev}\left(\pi_{i i}^{i}(a, s)\right)=0
$$

or this expression is strictly positive and

$$
\delta^{i} \leq \frac{-\operatorname{mev}\left(\pi_{i i}^{i}(a, s)\right)}{\left(\pi_{u}^{i}-\pi_{l}^{i}\right) \sum_{s^{\prime} \in S} \max \left\{0, \operatorname{mev}\left(Q_{i i}\left(s^{\prime} ; a, s\right)\right)\right\}-\operatorname{mev}\left(\pi_{i i}^{i}(a, s)\right)} .
$$

The following result provides a sufficient condition for a game to have convex best replies.

Proposition 2 Suppose that the game has concave reduced payoffs. Then, for all $i \in I$, all $a \in A$, all $s \in S$, and all function $v^{i}: S \rightarrow\left[\frac{\pi_{l}^{i}}{1-\delta^{i}}, \frac{\pi_{u}^{i}}{1-\delta^{i}}\right], \Pi^{i}\left(a, s ; v^{i}\right)$ is concave in $a^{i}$. In particular, the game has convex best replies.

This result provides a condition under which $\Pi^{i}\left(a, s ; v^{i}\right)$ is a concave function of $a^{i}$ for all $a^{-i} \in A^{-i}$, all $s \in S$, and all $v^{i}$. To gain intuition, suppose first that $\pi^{i}(a, s)$ is strictly concave in $a^{i}$. Then, even if for some $v^{i}$ the term $\sum_{s^{\prime} \in S} v^{i}\left(s^{\prime}\right) Q\left(s^{\prime} ; a, s\right)$ is ill-behaved (in the sense that it is "very convex"), the sum of $\pi^{i}(a, s)$ and $\delta^{i} \sum_{s^{\prime} \in S} v^{i}\left(s^{\prime}\right) Q\left(s^{\prime} ; a, s\right)$ can still be a concave function if $\delta^{i}$ is small enough. More generally, taking the maximum eigenvalue of the Hessian matrix as a measure of the concavity of a function (as we discussed above), (3.2) can be seen as making explicit a tension between the discount factor $\delta^{i}$ and the second derivatives with respect to $a^{i}$ of $\pi^{i}(a, s)$ and $Q\left(s^{\prime} ; a, s\right)$.

Proposition 2 establishes a trade-off between $\delta^{i}$ and the concavity of $\pi^{i}(a, s)$ and $Q\left(s^{\prime} ; a, s\right)$ as functions of $a^{i}$. Moreover, $\delta^{i}$ can be made arbitrarily close to 1 provided $Q\left(s^{\prime} ; a, s\right)$ is sufficiently flat or $\pi^{i}(a, s)$ is sufficiently concave in $a^{i}$. Indeed, as the following result shows, in some models Condition 3.2 imposes no restriction on $\delta^{i}$.

Lemma 3 Suppose that the for all $i, \pi^{i}(a, s)$ is a concave function of $a^{i}$, and that the transition function can be written as

$$
Q\left(s^{\prime} ; a, s\right)=\sum_{k=1}^{K} \alpha_{k}(a) F_{k}\left(s^{\prime} ; s\right)
$$

where for all $s, F_{k}(\cdot ; s)$ is a probability distribution over $S$ and, for all $a \in A, \sum_{k=1}^{K} \alpha_{k}(a)=1$ with $\alpha_{k}(a) \geq 0$ for all $k$. Assume that for all $k, \alpha_{k}$ is twice continuously differentiable as a function of $a^{i} \in A^{i} \subseteq \mathbb{R}^{L^{i}}$ and its second derivative equals 0. Then, the game has concave reduced payoffs and therefore best replies are convex. 
The importance of this lemma is that it provides easy to check sufficient conditions ensuring the convexity of the best replies. In particular, when the transition function $Q$ is a multilinear function of $\left(a^{1}, \ldots, a^{|I|}\right)$, the game has concave reduced payoffs provided each $\pi^{i}(a, s)$ is a concave function of $a^{i} \in A^{i}$. While in some applications this restriction on $Q$ may seem demanding, it provides as much flexibility in period payoffs as one can hope for (namely, concavity of payoffs as functions of own actions) and imposes no restriction on the discount factors.

One of the attractive features of games with concave reduced payoffs is their tractability. Indeed, in games with concave reduced payoffs, first order conditions, being necessary and sufficient for optimality, can be used to characterize equilibrium strategies. This observation is important not only when analytically deriving properties of the equilibrium strategies, but also when numerically solving for those strategies.

Let me now discuss how the restriction to games having concave reduced payoffs relates to similar conditions imposed in previous works. Horst's (2005) Weak Interaction Condition (2005) is strictly more demanding than the sufficient condition given above; this can be seen by noting that any stochastic game satisfying Condition (7) in Horst's (2005) paper also satisfies Condition (3.2).7 Indeed, Horst's (2005) assumption additionally makes reaction functions virtually flat functions of others' strategies. More recently, Nowak (2007) works under the assumption that $\pi^{i}(a, s)$ is concave in $a^{i}, Q\left(s^{\prime} ; a, s\right)$ is affine in $a^{i}$ (as in Lemma 3), and a strict diagonal dominance assumption holds.$^{8}$ It is not hard to see that under Nowak's (2007) concavity condition, $Q_{i i}=0$ and so the game has concave reduced payoffs and convex best replies for all $\delta^{i}<1$.

Amir (1996) and Curtat (1996) have studied supermodular stochastic games. These authors work under the assumption that the payoffs and the transition are supermodular and satisfy a positive spillovers property $!^{9}$ Moreover, these works still need to impose some

\footnotetext{
${ }^{7}$ To see this, assuming that $\pi_{l}^{i}=0, L^{i}=1$, and $\delta^{i}=\delta$ for all $i$, our condition 3.2 can be equivalently written as$$
\frac{\delta}{1-\delta}\left\|\pi^{i}\right\|_{\infty} \sup _{a \in A} \sum_{s^{\prime} \in S} \frac{\max \left\{0, Q_{i i}\left(s^{\prime} ; a, s\right)\right\}}{\left|\pi_{i i}^{i}(a, s)\right|} \leq 1
$$

for all $i$ and all $s$. Denoting by $\pi_{i j}^{i}(a, s)=\frac{\partial \pi^{i}(a, s)}{\partial a^{i} \partial a^{j}}$, Condition 7 on Assumption 2.2 in Horst (2005) can be written as

$$
\sum_{j \neq i} \sup _{a \in A} \frac{\left|\pi_{i j}^{i}(a, s)\right|}{\left|\pi_{i i}^{i}(a, s)\right|}+\frac{\delta}{1-\delta}\left\|\pi^{i}\right\|_{\infty} \sum_{j \neq i} \sup _{a \in A} \frac{\sum_{s^{\prime} \in S}\left|Q_{i j}\left(s^{\prime} ; a, s\right)\right|}{\left|\pi_{i i}^{i}(a, s)\right|}+\frac{\delta}{1-\delta}\left\|\pi^{i}\right\|_{\infty} \sup _{a \in A} \sum_{s^{\prime} \in S} \frac{\left|Q_{i i}\left(s^{\prime} ; a, s\right)\right|}{\left|\pi_{i i}^{i}(a, s)\right|}<1
$$

for all $i$ and all $s$. It follows that the left hand side of my restriction is strictly less than the left hand side of the restriction above.

${ }^{8}$ This assumption makes reaction functions in the static one-shot game a contraction.

${ }^{9} \mathrm{~A}$ game has positive spillovers if payoff functions are nondecreasing in rivals' actions.
} 
convexity restrictions. Consider, for example, Curtat's (1996) Strict Diagonal Dominance (SDD) assumption. To simplify the exposition, assume first that for all $i, A^{i} \subset \mathbb{R}$. Then, the SDD condition can be expressed as follows: For all $i$ and all $(a, s) \in A \times S, \sum_{i \in I} \frac{\partial^{2} \pi^{i}(a, s)}{\partial a^{i} \partial a^{j}}<0$. Since $\pi^{i}$ is supermodular, SDD implies that $\pi^{i}$ is strictly concave in $a^{i}$. More generally, if $A^{i} \subset \mathbb{R}^{L^{i}}, L^{i} \neq 1$, SDD is related to the concavity of $\pi^{i}(a, s)$ in $a$, but neither condition implies the other. Yet, the SDD condition on the transition restricts the model dynamics substantially. Indeed, in all the examples studied by Curtat (1996), the transition is a linear function of the action profile $a \in A$.

It is possible to state a number of corollaries by combining Theorem 1 and the sufficient conditions presented. The following corollaries will prove useful in applications.

Corollary 4 Let the game be transition continuous. If for all $i, \pi^{i}(a, s)$ is continuous in a, then the game possesses a Markov perfect equilibrium provided it has single valued best replies.

Corollary 5 Let the game be transition continuous game. If for all $i, \pi^{i}(a, s)$ is weak lower semi continuous, then the game possesses a Markov perfect equilibrium provided it has concave reduced payoffs.

By explicitly including time as a state variable, our framework encompasses alternating move games (Maskin and Tirole 1988), as well as finite horizon games. Additionally, we can allow the payoff functions and transition probabilities to depend not only on the current public signal but also on past signals just by redefining the state variable.

Our main result can also be applied to deduce the existence of behavior Markov perfect equilibria.

Theorem 6 Let the game be transition continuous, and $\pi^{i}(a, s)$ be upper semi continuous in $a \in A$ and lower semi continuous in $a^{-i} \in A^{-i}$. Then, it possesses a behavior Markov perfect equilibrium.

We observe that under the assumptions of the theorem above, $\pi^{i}(a, s)$ is continuous in $a^{-i}$. So, the theorem only allows for discontinuities in $a^{i}$. 


\subsection{Proof of Theorem 1}

In this subsection Theorem 1 is proven. The reader interested in applications may skip to Section 4. We begin by stating the following key result (Stokey and Lucas 1989, Theorem $9.2)$.

Lemma 7 For each $i \in I$, consider a function $\bar{a}^{i}: S \rightarrow A^{i}$. Suppose that there exists a tuple $\left(v^{i}\right)_{i \in I}$, where $v^{i}: S \rightarrow \mathbb{R}$ is bounded, such that for all $i$ and for all $s \in S$

$$
v^{i}(s)=\max \left\{\pi^{i}\left(\left(x^{i}, \bar{a}^{-i}(s)\right), s\right)+\delta^{i} \sum_{s^{\prime} \in S} v^{i}\left(s^{\prime}\right) Q\left(s^{\prime} ;\left(x^{i}, \bar{a}^{-i}(s)\right), s\right) \mid x^{i} \in A^{i}\right\}
$$

and

$$
\bar{a}^{i}(s) \in \arg \max \left\{\pi^{i}\left(\left(x^{i}, \bar{a}^{-i}(s)\right), s\right)+\delta^{i} \sum_{s^{\prime} \in S} v^{i}\left(s^{\prime}\right) Q\left(s^{\prime} ;\left(x^{i}, \bar{a}^{-i}(s)\right), s\right) \mid x^{i} \in A^{i}\right\} .
$$

Then, $\left(\bar{a}^{i}\right)_{i \in I}$ is a Markov perfect equilibrium.

This result allows us to reduce the problem of finding an equilibrium to the problem of solving a system of functional equations. We will therefore verify the existence of solutions to this system of functional equations using Kakutani's fixed point theorem.

For each $i$, define $\mathcal{A}^{i}$ as the set of functions $a^{i}: S \rightarrow A^{i}$ and $\mathcal{V}^{i}$ as the set of functions $v^{i}: S \rightarrow\left[\frac{\pi_{l}^{i}}{1-\delta^{i}}, \frac{\pi_{u}^{i}}{1-\delta^{i}}\right]$. Each of these sets is contained in a vectorial space that, when endowed with the product topology, is a Hausdorff topological vector space. Since $A^{i}$ and $\left[\frac{\pi_{l}^{i}}{1-\delta^{i}}, \frac{\pi_{u}^{i}}{1-\delta^{i}}\right]$ are compact, Tychonoff's theorem (Royden 1968, Chapter 9, Theorem 14) implies that $\mathcal{A}^{i}$ and $\mathcal{V}^{i}$ are compact. Additionally, since $S$ is countable, $\mathcal{A}^{i}$ and $\mathcal{V}^{i}$ are metric spaces (Royden 1968, Chapter 8, Exercise 45) 10 We define $\mathcal{A}=\prod_{i \in I} \mathcal{A}^{i}$, and $\mathcal{V}=\prod_{i \in I} \mathcal{V}^{i}$. We have therefore shown the following result.

Lemma $8 \mathcal{A} \times \mathcal{V}$ is a convex compact subset of a linear metric space.

\footnotetext{
${ }^{10}$ Observe that if $S$ were not countable, the sets $\mathcal{A}^{i}$ and $\mathcal{V}^{i}$ need not be metric spaces. The fact that these sets are metric spaces is used in the proof of Lemma 11.
} 
Now, for $i \in I$, consider the map $\Phi^{i}$ defined on $\mathcal{A} \times \mathcal{V}$ by

$$
(a, v) \in \mathcal{A} \times \mathcal{V} \mapsto \Phi^{i}(a, v)=\left\{\bar{a}^{i} \in \mathcal{A} \mid \bar{a}^{i}(s) \in \arg \max _{x^{i} \in A^{i}} \Pi^{i}\left(\left(x^{i}, a^{-i}(s)\right), s ; v^{i}\right) \text { for all } s \in S\right\}
$$

This map yields the best Markov strategies $\bar{a}^{i}$ for firm $i$, given arbitrary strategies for $i$ 's rivals and given continuation values $v^{i}$. Observe that the arguments $\left(v^{j}\right)_{j \neq i}$ and $a^{i}$ in the definition of $\Phi^{i}$ appear only for consistency (as will be shown soon).

It will also be useful to nail down continuation values. To do that, define the map $T^{i}$ by

$$
(a, v) \in \mathcal{A} \times \mathcal{V} \mapsto T^{i}(a, v)=\left\{\bar{v}^{i} \in \mathcal{V}^{i} \mid \bar{v}^{i}(s)=\max _{x^{i} \in A^{i}} \Pi^{i}\left(\left(x^{i}, a^{-i}(s)\right), s ; \bar{v}^{i}\right) \text { for all } s \in S\right\}
$$

This map yields the solutions to the dynamic programming problem faced by firm $i$, given the Markov strategies followed by $i$ 's rivals. Again, the dependance of this map on $\left(v^{j}\right)_{j \in I}$ and $a^{i}$ is just for consistency.

Finally define $\Phi$ by $\Phi(a, v)=\left(\prod_{i \in I} \Phi^{i}(a, v)\right) \times\left(\prod_{i \in I} T^{i}(a, v)\right)$. We state three preliminary results.

Lemma $9 \Phi(\mathcal{A} \times \mathcal{V}) \subseteq \mathcal{A} \times \mathcal{V}$

To see this lemma, fix $i$ and note that for any $(a, v) \in \mathcal{A} \times \mathcal{V}, \Phi^{i}(a, v) \subseteq \mathcal{A}^{i}$ and $T^{i}(a, v) \subseteq \mathcal{V}^{i}$. This implies that $\Phi(a, v) \subseteq \mathcal{A} \times \mathcal{V}$

Lemma $10 \Phi$ is nonempty- and convex-valued.

The proof of this lemma is as follows. Since the product of sets which are nonemptyand convex-valued inherits these properties, it is enough to prove that for each $i, \Phi^{i}$ and $T^{i}$ are nonempty- and convex-valued. Fix $i$. Given $a^{-i}: S \rightarrow A^{-i}$ and a continuation value $v^{i}: S \rightarrow \mathbb{R}_{+}$, for each $s$ the existence of solution for firm $i$ 's static problem 3.4 is evident; let $a_{i}(s)$ be such a solution. By definition, $a_{i} \in \Phi_{i}$ so that $\Phi_{i}$ is nonempty-valued. Moreover, $\Phi^{i}$ is convex-valued. Indeed, fix $\lambda \in[0,1]$ and consider $\phi^{i}, \phi^{i^{\prime}} \in \Phi^{i}(a, v)$, where $a, v \in \mathcal{A} \times \mathcal{V}$. Then, for all $s, \phi^{i}(s), \phi^{i^{\prime}}(s) \in \arg \max _{x^{i} \in A^{i}} \Pi^{i}\left(\left(x^{i}, a^{-i}(s)\right), s ; v^{i}\right)$. Since the game has convex best replies, for all $s, \lambda \phi^{i}(s)+(1-\lambda) \phi^{i^{\prime}}(s) \in \arg \max _{x^{i} \in A^{i}} \Pi^{i}\left(\left(x^{i}, a^{-i}(s)\right), s ; v^{i}\right)$. This implies that $\lambda \phi^{i}+(1-\lambda) \phi^{i^{\prime}} \in \Phi^{i}(a, v)$ and proves that $\Phi^{i}$ is convex-valued. Now, let us analyze $T^{i}$. Given $a^{-i}: S \rightarrow A^{-i}$, Theorem 13 in the Appendix implies the existence of a single function $v^{i} \in \mathcal{V}^{i}$ satisfying the dynamic programming condition 3.3 for firm $i$. Consequently, $T^{i}$, being the set of all such solutions to (3.3), is nonempty- and single-valued. This completes the proof. 
Lemma 11 Q has closed graph.

Since $\mathcal{A} \times \mathcal{V}$ is a metric space, to prove this lemma it is enough to prove that for any sequence $\phi_{n} \in \Phi\left(a_{n}, v_{n}\right)$ with $\phi_{n} \rightarrow \phi$ and $\left(a_{n}, v_{n}\right) \rightarrow(a, v)$, we have $\phi \in \Phi(a, v)$. Since the game is upper semi continuous, weak lower semi continuous, and transition continuous, Lemma 11 follows as an immediate consequence of Proposition 14 in the Appendix.

We are now in a position to provide a proof of Theorem 1. Lemma 8 implies that $\mathcal{A} \times \mathcal{V}$ is a compact convex set contained in a metric linear space. From Lemmata 9 , 10 and 11, $\Phi: \mathcal{A} \times \mathcal{V} \rightarrow \mathcal{A} \times \mathcal{V}$ is nonempty- and convex-valued, and its graph is closed. From Glicksberg's (1952) generalization of Kakutani's fixed point theorem, we deduce the existence of a fixed point $(\bar{a}, v) \in \mathcal{A} \times \mathcal{V}$ of $\Phi:(\bar{a}, v) \in \Phi(\bar{a}, v)$. It readily follows that, for each $i \in I$ and each $s \in S,(\bar{a}, v)$ satisfies conditions $(3.3)$ and (3.4). Lemma 7 implies that $\bar{a}$ is a Markov perfect equilibrium.

\section{Applications}

This section provides a number of applications of our main results. Subsection 4.1 studies a model similar to Ericson and Pakes's (1995) and relates our sufficient conditions to those recently derived by Doraszelski and Satterthwaite (2010). Subsection 4.2 shows a dynamic version of the textbook Cournot game with stochastic demand. Subsection 4.3 ensures equilibrium existence in a dynamic model of incomplete information (Bajari, Benkard, and Levin 2007, Doraszelski and Escobar 2010). Finally, Subsection 4.4 ensures existence in behavior strategies in a Markov Cournot game with fixed costs.

\subsection{Ericson-Pakes Industry Dynamics Model}

We now study an industry dynamics game in the spirit of Ericson and Pakes's (1995) seminal model. Consider a finite set $I$ of firms. At each $t$, some of the firms are incumbent; the others are entrant. The state of firm $i$ is $s^{i}=\left(\bar{s}^{i}, \eta^{i}\right) \in \bar{S}^{i} \times\{0,1\}$, where $\bar{s}^{i}$ reflects a demand or technology shock; $\eta^{i}=1$ if firm $i$ is an incumbent, and $\eta^{i}=0$ if firm $i$ is an entrant. The state of the industry is $s=\left(s^{i}\right)_{i \in I}$.

The action of firm $i$ is $\left(\mathbb{1}^{i}, x^{i}\right) \in\{0,1\} \times X$, with $X \subset \mathbb{R}_{+}^{L}$, where $\mathbb{1}^{i}=1$ (resp. $\mathbb{1}^{i}=0$ ) if firm $i$ changes (resp. does not change) its incumbency/entrance status and $x^{i}$ is a vector of investment projects firm $i$ undertakes. In other words, if firm $i$ is an entrant (resp. 
incumbent) and $\mathbb{1}^{i}=1$, then $i$ becomes an incumbent (resp. entrant). Since the set $\{0,1\}$ is not convex, we allow firms to randomize. Let $p^{i} \in[0,1]$ be the probability with which $i$ changes its statues. Firm $i$ 's action is therefore a vector $a^{i}=\left(p^{i}, x^{i}\right) \in[0,1] \times X$; we assume that $X$ is convex and compact.

Given a state $s$ and an action profile $a=\left(a^{i}\right)_{i \in I}$, the per period payoff for firm $i$ is given by

$$
\pi^{i}(a, s)=\eta^{i}\left(g^{i}(s)+\psi^{i}(s) p^{i}\right)+\left(1-\eta^{i}\right)\left(-\bar{\psi}^{i}(s) p^{i}\right)-c^{i}\left(x^{i}, s\right) .
$$

The first term is the profit that firm $i$ obtains when competing in a spot market, $g^{i}(s)$, plus the scrap value at which firm $i$ may be sold, $\psi^{i}(s)$, times the probability of exit $p^{i}$ when firm $i$ is incumbent, $\eta^{i}=1$. The second term is the setup price firm $i$ must pay to enter into the market, $-\bar{\psi}^{i}(s)$, times the probability of entry, $p^{i}$ when firm $i$ is an entrant, $\eta^{i}=0$. The third term is the cost of investment $x^{i}$ when the state is $s$. In applied work, one would restrict $g^{i}(s)$ to depend not on the whole vector $s$ but only on those $s^{j}$ for which firm $j$ is an incumbent $\eta^{j}=1$. Analogously, the scrap and set up values will typically depend only on the firm's own state $s^{i}$. Firm $i$ 's discount factor is $\delta^{i}$.

For a given vector $\mathbb{1}=\left(\mathbb{1}_{i}\right)_{i \in I} \in\{0,1\}^{|I|}$ of decisions on status changes and a profile $x=$ $\left(x^{i}\right)_{i \in I}$ of investment decisions, the state of the system in the following period is distributed according to $Q^{r}(\cdot ; \mathbb{1}, x, s)$. It is relatively easy to see that given the vector of actions $a=$ $(p, x)$, the next period state is distributed according to

$$
Q\left(s^{\prime} ; a, s\right)=\sum_{\mathbb{1} \in\{0,1\}^{|I|}} Q^{r}\left(s^{\prime} ; \mathbb{1}, x, s\right)\left(\prod_{j=1}^{|I|}\left(p^{j}\right)^{\mathbb{1}^{j}}\left(1-p^{j}\right)^{1-\mathbb{1}^{j}}\right)
$$

where we define $0^{0}=0$. We assume that $c^{i}\left(x^{i}, s\right)$ and $Q^{r}\left(s^{\prime} ; \mathbb{1}, x, s\right)$ are twice continuously differentiable functions of $x^{i}$.

A similar model is studied by Doraszelski and Satterthwaite (2010). They introduce the Unique Investment Choice (UIC) condition, a condition implying that the best reply set (3.1) is unique. It is therefore evident that after introducing a UIC condition in our model, the stochastic game has convex best replies and so the existence of equilibrium is a consequence of Corollary 4$]^{11}$ While the UIC condition may be applied to many variations of the EricsonPakes model, we provide an alternative condition that imposes much less structure on the

\footnotetext{
${ }^{11}$ For completeness, let me simplify the model to offer a UIC condition in the spirit of Doraszelski and Satterthwaite (2010). Suppose that the status change decision is (payoff) irrelevant, that is, the only choice variable is $x^{i}$. Also suppose that $X=[0,1]$ and $c^{i}\left(x^{i}, s\right)=x^{i}$. Then the UIC condition holds provided for all $i, Q\left(s^{\prime} ; x, s\right)=a^{i}\left(s^{\prime} ; x^{-i}, s\right) \eta^{i}\left(s, x^{i}\right)+b^{i}\left(s^{\prime} ; x^{-i}, s\right)$, where $\eta^{i}$ is twice differentiable, strictly increasing, and strictly concave in $x^{i}$. Under this condition, 3.1 is single valued.
} 
transition and the investment decisions.

First note that

$$
\pi_{i i}^{i}(a, s)=-c_{i i}^{i}\left(x^{i}, s\right)
$$

where $c_{i i}^{i}\left(x^{i}, s\right)$ denotes the matrix of second derivatives with respect to $x^{i}$. Now, the Hessian matrix of the transition function, $Q$, can be expressed as

$$
\begin{aligned}
Q_{i i}\left(s^{\prime} ; p, x, s\right)= & \sum_{\mathbb{1} \in\{0,1\}^{|I|}}\left(\prod_{j \neq i}\left(p^{j}\right)^{\mathbb{1}^{j}}\left(1-p^{j}\right)^{1-\mathbb{1}^{j}}\right) \times \\
& \left(\begin{array}{cc}
0 & {\left[Q_{x^{i}}^{r}\left(s^{\prime} ; \mathbb{1}, x, s\right)\right]^{\prime}\left(2 \mathbb{1}^{i}-1\right)} \\
Q_{x^{i}}^{r}\left(s^{\prime} ; \mathbb{1}, x, s\right)\left(2 \mathbb{1}^{i}-1\right) & Q_{x^{i} x^{i}}^{r}\left(s^{\prime} ; \mathbb{1}, x, s\right)\left(p^{i}\right)^{\mathbb{1}^{i}}\left(1-p^{i}\right)^{1-\mathbb{1}^{i}}
\end{array}\right)
\end{aligned}
$$

where $Q_{x^{i}}^{r}\left(s^{\prime} ; \mathbb{1}, x, s\right)$ (resp. $Q_{x^{i} x^{i}}^{r}\left(s^{\prime} ; \mathbb{1}, x, s\right)$ ) denotes the column vector of derivatives (resp. matrix of second derivatives) of $Q^{r}\left(s^{\prime} ; \mathbb{1}, x, s\right)$ with respect to the variable $x^{i}$. Denoting $\lambda^{i}\left(s^{\prime} ; p, x, s\right)=\operatorname{mev}\left(Q_{i i}\left(s^{\prime} ; p, x, s\right)\right)$, it follows that the Ericson-Pakes industry dynamics model has an equilibrium provided

$$
\delta^{i} \leq \frac{-\operatorname{mev}\left(-c_{i i}^{i}\left(x^{i}, s\right)\right)}{\mu^{i} \sum_{s^{\prime} \in S} \max \left\{0, \lambda^{i}\left(s^{\prime} ; p, x, s\right)\right\}-\operatorname{mev}\left(-c_{i i}^{i}\left(x^{i}, s\right)\right)}
$$

for all $i, p, x$ and $s$, where $\mu^{i}=\pi_{u}^{i}-\pi_{l}^{i}$ equals

$$
\mu^{i}=\max _{s^{\prime \prime} \in S \text { with } \eta^{i}=1, y^{i} \in X}\left(g^{i}\left(s^{\prime \prime}\right)+\psi^{i}\left(s^{\prime \prime}\right)-c^{i}\left(y^{i}, s^{\prime \prime}\right)\right)+\max _{s^{\prime \prime} \in S \text { with } \eta^{i}=0, y^{i} \in X}\left(\bar{\psi}^{i}\left(s^{\prime \prime}\right)+c^{i}\left(y^{i}, s^{\prime \prime}\right)\right) .
$$

While (4.1) is not more general than the UIC condition (a condition already shown to fit into our general framework), this new condition allows modeling alternatives uncovered by Doraszelski and Satterthwaite (2010). Doraszelski and Satterthwaite's (2010) analysis hinges on the unidimensionality of the investment decisions -ruling out, for example, investment plans that can affect the demand and the cost structure independently-, and the separable form of the transition - ruling out several transitions exhibiting non trivial complementarities among the investment decisions. This and other modeling alternatives can be analyzed with this new alternative condition.

Condition 4.1) involves the maximum eigenvalue of the matrix of second derivatives of minus the cost function. Intuitively, the condition says that $-c_{i i}^{i}$ must be sufficiently concave, given the discount factor $\delta^{i}$, so that all of its eigenvalues are negative enough. Alternatively, the firm must be sufficiently impatient given the technology $c_{i i}^{i}$. Condition 4.1 resonates well with other existence results in equilibrium theory, which emphasize the importance of ruling out increasing returns of production to ensure equilibrium existence (Mas-Colell, Whinston, 
and Green 1995, Proposition 17.BB.2). The novel aspect of Condition (4.1) is that, because of the dynamics, convexity of the cost functions must be strengthened so that, even when firms maximize total payoffs, best replies are convex valued. To do this, we restrict attention to models in which returns to scale are "sufficiently decreasing".

It is also of interest to observe that when 4.1 holds, each firm's payoff is a concave function of its decision variables. Thus, first order conditions are necessary and sufficient for optimality. The problem of numerically finding equilibrium strategies is therefore effectively reduced to the problem of solving a (potentially huge) system of equalities (or variational inequalities). Standard numerical methods -as the homotopy method implemented by Besanko, Doraszelski, Kryukov, and Satterthwaite (2010)- are rarely ensured to converge. Remarkably, under (4.1), we can be confident that a converging method will yield an equilibrium of the model.

In applications, checking Condition (4.1) amounts to solving $|I| \times|S|$ nonlinear minimization problems on $([0,1] \times X)^{|I|}$. In complicated models, this can be done numerically before running the routines to solve for the equilibria. This first step is relatively easy to implement numerically as the $|I| \times|S|$ minimization problems are unrelated. If this initial step is successful, our model is well behaved in that not only it possesses an equilibrium but also all the dynamic programming problems involved will be concave maximization problems.

The following example presents a simple model in which investment decisions are multidimensional and returns to scale are decreasing; we observe that Doraszelski and Satterthwaite's (2010) results do not apply.

Example 12 Suppose that firms invest jointly in a project and the total investment determines the common state of the industry $\bar{s}$. In other words, we now assume that $\bar{s}^{i}=\bar{s}^{j}$ for all $i \neq j$, and that this state is stochastically determined by $\sum_{i=1}^{|I|} x^{i}$, where $x^{i} \in[0,1]^{2}$. The state $\bar{s}$ only determines spot market profits so that the profit of a firm competing in the spot market is $g(\bar{s}, \eta)$, while scrap values and set up prices are $\psi^{i}(s)=\psi \in \mathbb{R}_{+}$and $\bar{\psi}^{i}(s)=\bar{\psi} \in \mathbb{R}_{+}$for all $i$ and all $s$. Each firm may carry out two types of investment projects so that $x^{i} \in[0,1]^{2}$, and the cost functions take the form $c^{i}\left(x^{i}, s\right)=\frac{1}{2}\left(\left(x_{1}^{i}\right)^{2}+\left(x_{2}^{i}\right)^{2}\right)$. We assume that $\delta^{i}=\delta$ for all $i$. This is a symmetric model in which firms jointly invest in improving spot market profits for example, by doing advertising or by making the (non proprietary) production technology more efficient. We refer to the state $\bar{s}$ as the spot market conditions.

The set of spot market conditions $\bar{S}$ is ordered and can be written $\bar{S}=\{1, \ldots,|\bar{S}|\}$. Higher states result in higher profits so that $g(\bar{s}, \eta)$ is increasing in $\bar{s}$. The evolution of the 
spot market conditions $\bar{s}$ takes the form

$$
Q^{r}\left(\bar{s}^{\prime} ; x, \bar{s}\right)=\alpha\left(\sum_{i=1}^{|I|} x^{i}\right) F_{1}\left(\bar{s}^{\prime} ; \bar{s}\right)+\left(1-\alpha\left(\sum_{i=1}^{|I|} x^{i}\right)\right) F_{2}\left(\bar{s}^{\prime} ; \bar{s}\right)
$$

where $\alpha(y) \in[0,1]$ for all $y \in[0,|I|]^{2}$, and $F_{k}(\cdot \mid \bar{s})$ is a probability distribution over $\bar{S}$ (as in Lemma 3). The transitions are so that $F_{1}$ moves the subsequent state up deterministically in one step (or stays in the same state if the current step is $|\bar{S}|$ ), while $F_{2}$ moves the state down in one step (or stays in the same state if the current step is 1). Intuitively, the highest the joint effort $y=\sum_{i \in I} x^{i}$, the more likely the next spot market conditions will be favorable. We assume that $\alpha(\cdot)$ is a linear function and that the first dimension of the investments is more effective: $\alpha(y)=\frac{1}{2}+\alpha_{1} y_{1}+\alpha_{2} y_{2}$ with $\alpha_{2}=\alpha_{1} / 2$. If no firm invests, then the subsequent state is equally likely to go up or down.

Whether firms enter or exit the market is determined by

$$
q\left(\eta^{\prime} ; p, \eta\right)=\left(\prod_{i: \eta^{i}=\eta^{\prime}} p^{i}\right)\left(\left(\prod_{i: \eta^{i} \neq \eta^{\prime i}}\left(1-p^{i}\right)\right)\right.
$$

Therefore the transition takes the form

$$
Q\left(s^{\prime} ; p, x, s\right)=\left(\prod_{j: \eta^{j}=\eta^{\prime j}} p^{j}\right)\left(\prod_{j: \eta^{j} \neq \eta^{\prime}}\left(1-p^{j}\right)\right) \cdot Q^{r}\left(\bar{s}^{\prime} ; x, s\right) .
$$

Once we have an expression for the transition function, it is relatively easy to show that ${ }^{12}$

$$
\sum_{s^{\prime} \in S} \max \left\{0, \lambda^{i}\left(s^{\prime} ; p, x, s\right)\right\}=2 \sqrt{\alpha_{1}^{2}+\alpha_{2}^{2}}=\sqrt{5} \alpha_{1}
$$

and that $\operatorname{mev}\left(-c_{i i}^{i}\left(x^{i}, s\right)\right)=-1$. We also assume that $\bar{g}+\psi+\bar{\psi}=1$, where $\bar{g}=\max _{s} g(s)$, meaning that the sum of spot market profits, scrap values, and set up costs is at most 1 (which is the maximum investment cost a firm can incur). We derive the following sufficient

${ }^{12}$ To see this, note that $Q_{i i}$ takes the form

$$
P\left[\eta_{-i}^{\prime} \mid \eta_{-i}, p\right]\left(F_{1}\left(\bar{s}^{\prime} ; \bar{s}\right)-F_{2}\left(\bar{s}^{\prime} ; \bar{s}\right)\right)\left(\begin{array}{ccc}
0 & \alpha_{1} & \alpha_{2} \\
\alpha_{1} & 0 & 0 \\
\alpha_{2} & 0 & 0
\end{array}\right)
$$

and therefore $\operatorname{mev}\left(Q_{i i}\left(s^{\prime} ; p, x, s\right)\right)=P\left[\eta_{-i}^{\prime} \mid \eta_{-i}, p\right]\left|F_{1}\left(\bar{s}^{\prime} ; \bar{s}\right)-F_{2}\left(\bar{s}^{\prime} ; \bar{s}\right)\right| \sqrt{\alpha_{1}^{2}+\alpha_{2}^{2}}$. Noting that each $F_{k}(\cdot ; \bar{s})$ puts positive weight on 2 states and summing up over subsequent states $s^{\prime} \in S$, the result follows. 
condition for equilibrium existence:

$$
\delta \leq \frac{1}{2 \sqrt{5} \alpha_{1}+1}
$$

For example, if $\alpha_{1}=1 / 100$ (so that a firm investing $\Delta>0$ units can increase the probability of the high state in $\Delta$ per cent), then the condition above amounts to $\delta \leq .96{ }^{13}$

When firms make entry and exit decisions before investment decisions, and when making investment decisions firms observe the identity of the market participants, then in the model above the existence of Markov perfect equilibrium can be guaranteed using Lemma 3. regardless of the discount factor. In such a model, since the transition $Q^{r}$ is linear in the investment decisions and entry and exit decisions are randomized strategies, the game has concave reduced payoffs for all discount factors. In some industries, advertising decisions are likely to take place after the identity of the market participants is publicly known, and therefore a model of sequential decisions would seem more appropriate. Of course, what the appropriate timing is will depend on the application.

\subsection{Markov Cournot Oligopoly}

We now consider a simple dynamic version of the textbook Cournot game. There is a finite set $I$ of oligopolists. At each $t$, oligopolists set quantities $a_{t}^{i} \in[0,1], i \in I$, simultaneously and independently. The (inverse) demand function takes the form $P\left(\sum_{i \in I} a_{t}^{i}, s_{t}\right)$, where the state, $s_{t}$, belongs to a finite set. There are no costs of production. So, the period payoff to firm $i$ is

$$
\pi^{i}(a, s)=a_{i} \cdot P\left(\sum_{i \in I} a^{i}, s\right) .
$$

The demand function assumes the functional form $P\left(\sum_{i \in I} a^{i}, s\right)=s \cdot\left(1-\sum_{i \in I} a^{i}\right)$. Players discount future payoffs at a constant rate $\delta \in] 0,1[$.

The set of states $S$ is a countable subset of $] 0, \infty\left[\right.$. The evolution of the state $s^{t}$ is given by the transition

$$
Q\left(s^{\prime} ; a, s\right)=\sum_{k=1}^{K} \alpha_{k}\left(\sum_{i \in I} a^{i}\right) F_{k}\left(s^{\prime} ; s\right)
$$

where $K$ is a finite number, $\alpha_{k}$ is a quadratic function of $\sum_{i \in I} a^{i}$, and $F_{k}(\cdot ; s)$ is a probability distribution on $S$. As previously discussed, we can interpret this transition as being drawn in

\footnotetext{
${ }^{13}$ Observe that since $\alpha(y)$ must belong to $[0,1], \alpha_{1}$ is bounded above by $\frac{1}{3|I|}$.
} 
two steps: first, we draw a lottery over the set $\{1, \ldots, K\}$ (where the weights are determined by the total production $\left.\sum_{i \in I} a^{i}\right)$, then, given the result $k$ of the lottery, a draw from the distribution $F_{k}(\cdot ; s)$ is realized and determines the subsequent state. The assumption that $\alpha_{k}$ is a quadratic function of $\sum_{i \in I} a^{i}$ implies that its second derivative is constant; let $\bar{\alpha}_{k}$ be the second derivative of $\alpha_{k}$. We also assume that $F_{k}(\cdot ; s)$ puts weight 1 on some state $s^{\prime} \in S$ (for example, it may put weight 1 on the state immediately below or above $s$ ).

It is relatively easy to see that ${ }^{14}$

$$
\sum_{s^{\prime} \in S} \max \left\{0, Q_{i i}\left(s^{\prime} ; a, s\right)\right\} \leq \sum_{k=1}^{K}\left|\bar{\alpha}_{k}\right|
$$

for all $a \in[0,1]^{|I|}$ and all $s \in S$. The existence of Markov perfect equilibrium is guaranteed provided

$$
\delta \leq \frac{1}{\frac{H}{4 L} \sum_{k=1}^{K}\left|\bar{\alpha}_{k}\right|+1}
$$

where $H=\max \{s \in S\}$ and $L=\min \{s \in S\}(>0)$. Note that the results by Curtat (1996) do not apply to this Cournot setting for he considers supermodular games satisfying strong monotonicity restrictions. To ensure existence we do not need to impose conditions on the number of players, nor do we need to assume that $\bar{\alpha}_{k}=0$ for all $k$. In order to apply Horst's (2005) and Nowak's (2007) results, regardless of the transition function, we would need to impose that $|I| \leq 2$. Moreover, results by Nowak (2007) can be applied only if we considered a linear transition.

For example, consider a model of habit formation, in which $K=2, \alpha_{1}\left(\sum_{i \in I} a^{i}\right)=$ $\bar{\alpha}_{1}\left(\sum_{i \in I} a^{i}\right)^{2}$, with $\bar{\alpha}_{1}>0$, and given $s \in S, F_{1}(\cdot ; s)$ puts weight 1 on a point strictly greater than the point in which $F_{2}(\cdot ; s)$ puts weight on. The idea is that the higher the volume of sales $\sum_{i \in I} a^{i}$, the higher the probability the next demand state $s^{\prime}$ will be high. Since $\alpha_{1}\left(\sum_{i \in I} a^{i}\right) \in[0,1]$ and $\alpha_{1}\left(\sum_{i \in I} a^{i}\right)+\alpha_{2}\left(\sum_{i \in I} a^{i}\right)=1$, it follows that $\left|\bar{\alpha}_{k}\right| \leq \frac{1}{|I|^{2}}$ for $k=1,2$. Assuming that $H / L=2$, the sufficient condition for equilibrium existence is

$$
\delta \leq \frac{|I|^{2}}{|I|^{2}+1}
$$

\footnotetext{
${ }^{14}$ To see this, note that $Q_{i i}\left(s^{\prime} ; a, s\right)=\sum_{k=1}^{K} \bar{\alpha}_{k} F_{k}\left(s^{\prime} ; s\right)$. Fix $s$, and let $s^{\prime}(k)$ be the state with weight 1 given $F_{k}(\cdot ; s)$. Then

$$
\sum_{s^{\prime} \in S} \max \left\{0, Q_{i i}\left(s^{\prime} ; a, s\right)\right\} \leq \sum_{s^{\prime} \in S} \sum_{k=1}^{K}\left|\bar{\alpha}_{k}\right| F_{k}\left(s^{\prime} ; s\right)=\sum_{k=1}^{K}\left|\bar{\alpha}_{k}\right| F_{k}(s(k) ; s)=\sum_{k=1}^{K}\left|\bar{\alpha}_{k}\right| .
$$
}


When $|I|=2$, there exists an equilibrium provided $\delta \leq \frac{4}{5}$, while when $|I|=6$ equilibrium existence is guaranteed when $\delta \leq \frac{36}{37}$.

\subsection{Dynamic Model with Incomplete Information}

We now consider a model of dynamic interaction with private information. Similar models are extensively employed in the applied literature; consult Bajari, Benkard, and Levin (2007) for a recent application.

Consider a model as that introduced in Section 2, but now suppose that at the beginning of each period, each firm not only observes the public state $s_{t}$ but also receives a private shock $\nu_{t}^{i} \in \mathbb{R}^{N_{i}}$. Then, each firm picks its action $a_{t}^{i}$ and gets a period profit $\pi^{i}\left(a, s, \nu^{i}\right)$. Private shocks are drawn independently according to a distribution function $G^{i}(\cdot), i \in I$, and the transition function takes the form $Q\left(s^{\prime} ; a, s\right)$.

A pure strategy for a firm is a function $\bar{a}^{i}: S \times \mathbb{R}^{N_{i}} \rightarrow A^{i}$. However, in order to apply our general framework, a strategy is interpreted as a function $\bar{a}^{i}: S \rightarrow \mathbb{A}^{i}$, where

$$
\mathbb{A}^{i}=\left\{a^{i}: \mathbb{R}^{N_{i}} \rightarrow A^{i} \mid a^{i} \text { is measurable }\right\}
$$

Functions in $\mathbb{A}^{i}$ are deemed identical if they are equal $G^{i}$-almost sure. Given functions $a^{i} \in \mathcal{A}^{i}$ and a public state $s \in S$, define

$$
\tilde{\pi}^{i}(a, s)=\int \pi^{i}\left(\left(a^{1}\left(\nu^{1}\right), \ldots, a^{I}\left(\nu^{I}\right)\right), s, \nu^{i}\right) G^{1}\left(d \nu^{1}\right) \ldots G^{I}\left(d \nu^{I}\right)
$$

and

$$
\widetilde{Q}\left(s^{\prime} ; a, s\right)=\int Q\left(s^{\prime} ;\left(a^{1}\left(\nu^{1}\right), \ldots, a^{I}\left(\nu^{I}\right)\right), s\right) G^{1}\left(d \nu^{1}\right) \ldots G^{I}\left(d \nu^{I}\right),
$$

This defines a dynamic game that fits into our dynamic stochastic game framework. Applications of this private information set up abound (Bajari, Benkard, and Levin 2007, Doraszelski and Escobar 2010).

To see the importance of the private shocks when applying our results, assume that $A^{i}$ is finite, $G^{i}$ is absolutely continuous with respect to the Lebesgue measure, $N^{i}=\left|A^{i}\right|$, and the period payoff function takes the form

$$
\pi^{i}\left(a, s, \nu^{i}\right)=g^{i}(a, s)+\sum_{k \in A^{i}} \mathbb{1}_{a^{i}=k} \nu^{i, k}
$$

Now, endow $A^{i}$ with the discrete topology and $\mathbb{A}^{i}$ with the convergence in measure metric. 
That is, given measurable functions $a^{i}, b^{i}: \mathbb{R}^{N^{i}} \rightarrow A^{i}$, define

$$
\left.d^{\mathrm{A}^{i}}\left(a^{i}, b^{i}\right)=\int \frac{d^{i}\left(a^{i}\left(\nu^{i}\right), b^{i}\left(\nu^{i}\right)\right)}{1+d^{i}\left(a^{i}\left(\nu^{i}\right), b^{i}\left(\nu^{i}\right)\right.}\right) G^{i}\left(d \nu^{i}\right) .
$$

where $d^{i}$ is the discrete metric over $A^{i}$. Under $d^{\mathbb{A}^{i}}, \mathbb{A}^{i}$ is compact. The transition $\widetilde{Q}\left(s^{\prime} ; a, s\right)$ is continuous and for all $i$, the payoff $\tilde{\pi}^{i}(a, s)$ is continuous in $a \in \mathbb{A}$. Private signals come crucially into play when verifying the convexity of the best replies. Indeed, it is not hard to see that the best reply set of each firm is (essentially) unique for any continuation value function ${ }^{15}$ So, the game has single-valued best replies and the existence of equilibrium follows from Corollary 4.

\subsection{Markov Cournot Oligopoly with Fixed Costs}

We finally apply our results to a Markov Cournot game with fixed costs. Fixed costs introduce discontinuities that make all previous results in the literature unapplicable ${ }^{16}$

There is a finite set $I$ of oligopolists that, at each $t$, set quantities $a^{i} \in[0,1], i \in I$, simultaneously and independently. The (inverse) demand function takes the form $P\left(\sum_{i \in I} a^{i}, s\right)$, where the state, $s$, belongs to a countable set $S . P\left(\sum_{i \in I} a^{i}, s\right)$ is a continuous function of $\sum_{i \in I} a^{i}$. Firm $i$ 's cost function, $c^{i}\left(a^{i}, s\right)$, is lower semi continuous in $a^{i}$. For example, suppose that each firm must incur a fixed $\operatorname{cost} \kappa>0$ to produce any (strictly) positive quantity, and that marginal costs equal 0 . Then, firm $i$ 's cost function can be written as

$$
c^{i}\left(a^{i}, s\right)= \begin{cases}0 & \text { if } a^{i}=0 \\ \kappa & \text { if } a^{i}>0\end{cases}
$$

This cost function is lower semi continuous at $a^{i}=0$. More generally, the presence of fixed costs (that, by definition, can be avoided if production is suspended in a given round) make cost functions naturally lower semi continuous, but not continuous, at $a^{i}=0$. Hence, the period payoff to firm $i$ is

$$
\pi^{i}(a, s)=a_{i} P\left(\sum_{i \in I} a^{i}, s\right)-c^{i}\left(a^{i}, s\right)
$$

\footnotetext{
${ }^{15}$ Indeed, a firm is indifferent between two actions with probability zero.

${ }^{16}$ This paper seems to be the first one guaranteeing existence in a nontrivial discontinuous dynamic setting. In this respect, the topological restrictions imposed by Theorem 1 are slightly more restrictive than those imposed by Dasgupta and Maskin (1986). The main difference, which does not seem relevant in applications, is that the lower semi continuity condition they impose is more permissive than ours.
} 
The transition is $Q$ is assumed setwise continuous in $a \in A$. Since $\pi^{i}$ is upper semi continuous in $a$ and lower semi continuous in $a^{-i}$, the existence of behavior strategy Markov perfect equilibrium follows from Theorem $6{ }^{17}$

\section{Concluding Comments}

I offer results guaranteeing existence of Markov perfect equilibrium in a class of dynamic stochastic games. Dynamic models that can be solved analytically are exceptional and therefore oftentimes researchers need to resort to computational routines to analyze their models. Yet, unless an equilibrium is guaranteed to exist, a non converging algorithm designed to compute an equilibrium may fail either because an equilibrium exists and the algorithm is not suitable for its computation or, more dramatically, because an equilibrium does not exist. The results in this paper provide guidance on the nature of dynamic models possessing Markov perfect equilibrium (in pure and behavior strategies). In doing so, we expand and unify several modeling alternatives available (Horst 2005, Doraszelski and Satterthwaite 2010) and apply our results to several dynamic models of imperfect competition. We impose restrictions on the fundamentals of the model ensuring the each firm's optimization problem has a concave objective function. This property not only constitutes a sufficient condition for equilibrium existence but also makes available numerical algorithms more reliable.

The upper semi continuity restriction we impose on the payoff functions limits the applicability of our results. As a consequence of this assumption, our setting does not permit applications to auction and pricing games. In fact, in those games the possibility of equilibrium ties makes payoff functions not upper semi continuous. Unless one rules out equilibrium ties, our results are not applicable to auction and pricing games. This is an important research avenue.

\footnotetext{
${ }^{17}$ As in static models, it is hard to ensure the existence of (pure) Markov perfect equilibria in models with fixed costs. Thus, the most we can hope for is probably behavior strategy existence.
} 


\section{Appendix}

\subsection{Omitted Proofs}

Proof of Proposition 2 Observe first that $\Pi_{i i}^{i}\left(a, s ; v^{i}\right)=\pi_{i i}^{i}(a, s)+\delta^{i} \sum_{s^{\prime} \in S} v^{i}\left(s^{\prime}\right) Q_{i i}\left(s^{\prime} ; a, s\right)$. Therefore, $\Pi_{i i}^{i}$ is concave if for all $x \in \mathbb{R}^{L^{i}}$,

$$
x^{\prime} \pi_{i i}^{i}(a, s) x+\delta^{i} \sum_{s^{\prime} \in S} v^{i}\left(s^{\prime}\right) x^{\prime} q_{i i}\left(s^{\prime} ; a, s\right) x \leq 0 .
$$

To prove this property, observe that for any symmetric matrix,

$$
x^{\prime} M x \leq \operatorname{mev}(M)\|x\|^{2} .
$$

Now, assume first that $\pi_{l}^{i}=0$ and therefore $v: S \rightarrow \mathbb{R}_{+}$. Then

$$
\begin{aligned}
x^{\prime} \pi_{i i}^{i}(a, s) x & +\delta^{i} \sum_{s^{\prime} \in S} v^{i}\left(s^{\prime}\right) x^{\prime} Q_{i i}\left(s^{\prime} ; a, s\right) x \\
& \leq \operatorname{mev}\left(\pi_{i i}^{i}(a, s)\right)\|x\|^{2}+\delta^{i} \frac{\pi_{u}^{i}}{1-\delta^{i}} \sum_{s^{\prime} \in S} \max \left\{0, x^{\prime} Q_{i i}\left(s^{\prime} ; a, s\right) x\right\} \\
& \leq \operatorname{mev}\left(\pi_{i i}^{i}(a, s)\right)\|x\|^{2}+\delta^{i} \frac{\pi_{u}^{i}}{1-\delta^{i}} \sum_{s^{\prime} \in S} \max \left\{0, \operatorname{mev}\left(Q_{i i}\left(s^{\prime} ; a, s\right)\right)\|x\|^{2}\right\} \\
& =\left(\operatorname{mev}\left(\pi_{i i}^{i}(a, s)\right)+\delta^{i} \frac{\pi_{u}^{i}}{1-\delta^{i}} \sum_{s^{\prime} \in S} \max \left\{0, \operatorname{mev}\left(Q_{i i}\left(s^{\prime} ; a, s\right)\right)\right\}\right)\|x\|^{2} \\
& \leq 0 .
\end{aligned}
$$

Under the conditions of the lemma, this expression is less than or equal to 0 . When $\pi_{l}^{i} \neq 0$, modify the payoffs and consider $\bar{\pi}^{i}(a, s)=\pi^{i}(a, s)-\pi_{l}^{i}$. Observe that the game with payoffs $\pi^{i}$ has convex best replies iff so does the game with payoffs $\bar{\pi}^{i}$. The lower bound for the function $\bar{\pi}^{i}$ is 0 and therefore the original dynamic game has convex best replies provided

$$
\operatorname{mev}\left(\pi_{i i}^{i}(a, s)\right)+\delta^{i} \frac{\pi_{u}^{i}-\pi_{l}^{i}}{1-\delta^{i}} \sum_{s^{\prime} \in S} \max \left\{0, \operatorname{mev}\left(Q_{i i}\left(s^{\prime} ; a, s\right)\right)\right\} \leq 0
$$

The result follows rearranging terms.

Proof of Theorem 6 We will define a new game and apply Theorem 1 to this new game. Denote the set of probability measures on $A^{i}$ by $\mathcal{P}\left(A^{i}\right)$. We endow the set $\mathcal{P}\left(A^{i}\right)$ with the weak* topology; consult Chapter 15 in Aliprantis and Border (2006) for details. This space 
is contained in a linear space. Given a profile $\mu=\left(\mu^{i}\right)_{i \in I} \in \prod_{i \in I} \mathcal{P}\left(A^{i}\right)$, we can define

$$
\bar{\pi}^{i}(\mu, s)=\int \pi^{i}(a, s)\left(\mu^{1} \otimes \cdots \otimes \mu^{|I|}\right)(d a)
$$

Analogously, we can extend the transition function defining

$$
\bar{Q}\left(s^{\prime} ; \mu, s\right)=\int Q\left(s^{\prime} ; a, s\right)\left(\mu^{1} \otimes \cdots \otimes \mu^{|I|}\right)(d a)
$$

Under our assumptions, these extensions are well defined. We can therefore consider a new dynamic stochastic game, called the extended game, in which player $i$ 's set of actions is $\mathcal{P}\left(A^{i}\right)$

and payoffs and transitions, $\bar{\pi}^{i}$ and $\bar{Q}$, are evaluated taking expectations. Observe that a Markov perfect equilibrium of the extended game is a behavior Markov perfect equilibrium of the original game. Since $A^{i}$ is compact, so is $\mathcal{P}\left(A^{i}\right)$ (Aliprantis and Border 2006, Theorem 15.11). It can be shown that since $Q\left(s^{\prime} ; a, s\right)$ is continuous in $a$ and $\pi^{i}(a, s)$ is upper semi continuous in $a$ and lower semi continuous in $a^{-i}$, the extensions inherit these properties (Aliprantis and Border 2006, Theorem 15.5). Since the extended game has convex best replies, it possesses a Markov perfect equilibrium which in turn yields the desired result.

\subsection{Dynamic Programming Results}

Consider the functional equation

$$
V(s)=\sup _{x \in X}\left\{\pi(x, s)+\delta \sum_{s^{\prime} \in S} V\left(s^{\prime}\right) \nu\left(s^{\prime} ; x, s\right)\right\}, \quad s \in S,
$$

where $X$ is a compact subset of a metric space, $\pi(x, s)$ is the per period profit function, $\delta \in[0,1[, \nu(\cdot ; x, s)$ is a probability distribution over $S$.

In this subsection the solutions to the functional equation (6.1) are studied. Results concerning the existence and continuity of those solutions are provided. Consider the following assumptions.

(D1) $\pi(x, s)$ is upper semi continuous in $x \in X$.

(D2) $\nu\left(s^{\prime} ; x, s\right)$ is setwise continuous in $x \in X$ : for all $E \subseteq S, \nu\left(s^{\prime} ; x, s\right)$ is continuous in $x \in X$

The following result guarantees the existence of a bounded solution for 6.1. 
Theorem 13 Assume (D1)-(D2). Then, there exists a single solution $\bar{V}$ to (6.1). Moreover, $\|\bar{V}\|_{\infty} \leq\|\pi\|_{\infty} /(1-\delta)$. Further, there exists a policy function $\bar{x}: S \rightarrow X$.

The proof of this result is standard. The idea is that the left hand side of 6.1, as a function of the continuation value function $V$, defines a contraction map. Since payoffs are bounded, the contraction mapping theorem (Stokey and Lucas 1989, Theorem 3.2) implies the existence and uniqueness of a solution $\bar{V}$. A similar method of proof is employed in Theorem 9.6 in Stokey and Lucas (1989).

Now, let us study continuity properties for the only solution to (6.1), viewing this solution as a function of the transition function $\nu$ and the per period payoff $\pi$. We can define

$$
T V(s)=\sup _{x \in X}\left\{\pi(x, s)+\delta \sum_{s^{\prime} \in S} V\left(s^{\prime}\right) \nu\left(s^{\prime} ; x, s\right)\right\}
$$

For each $n \in \mathbb{N}$, consider a transition function $\nu_{n}$ and a per period payoff function $\pi_{n}$. For each $n$, consider the operator $T_{n}$ defined as we did in (6.2), but replacing $\pi$ and $\nu$ with $\pi_{n}$ and $\nu_{n}$ respectively.

Let $\bar{V}_{n}$ and $\bar{V}$ be the only bounded functions such that $T_{n} \bar{V}_{n}=\bar{V}_{n}$ and $T \bar{V}=\bar{V}$. Additionally, let the set valued maps $\bar{X}_{n}$ and $\bar{X}$ be defined by

$$
\bar{X}_{n}(s ; V)=\arg \max _{x \in X}\left\{\pi_{n}(x, s)+\delta \sum_{s^{\prime} \in S} V\left(s^{\prime}\right) \nu_{n}\left(s^{\prime} ; x, s\right)\right\}
$$

and

$$
\bar{X}(s ; V)=\arg \max _{x \in X}\left\{\pi(x, s)+\delta \sum_{s^{\prime} \in S} V\left(s^{\prime}\right) \nu\left(s^{\prime} ; x, s\right)\right\} .
$$

The following result shows that the only solution to $(6.1)$ and the related policy map depends continuously on $\nu$ and $\pi$.

Proposition 14 For all $n \in \mathbb{N}$, assume (D1)-(D2) for the problems defined by $T_{n}$ and $T$.

Suppose that for all sequence $x_{n} \rightarrow x$ in $X, E \subseteq S$, and $s \in S$, the sequence of real numbers $\left(\nu_{n}\left(E ; x_{n}, s\right)\right)_{n \in \mathbb{N}}$ converges to $\nu(E ; x, s)$ Further suppose that for all $s \in S$

1. For all sequence $x_{n} \rightarrow x$ in $X$, $\limsup _{n \rightarrow \infty} \pi_{n}\left(x_{n}, s\right) \leq \pi(x, s)$;

2. For all $x \in X$, there exists $y_{n} \rightarrow x$ in $X$, such that $\liminf _{n \rightarrow \infty} \pi_{n}\left(y_{n}, s\right) \geq \pi(x, s)$.

Then, the following statements hold. 
(a) For all subsequence $V_{n_{j}} \rightarrow V$ (pointwise) and given any selection $\bar{x}_{n_{j}}(\cdot) \in \bar{X}_{n_{j}}\left(\cdot ; V_{n_{j}}\right)$ converging to $\bar{x}: S \rightarrow X, \bar{x}(s) \in \bar{X}(s ; V)$ for all $s \in S$.

(b) For all $s \in S, \bar{V}_{n}(s) \rightarrow \bar{V}(s)$.

(c) The policy sets are closed maps of the per period payoff and transition functions.

Before proving this proposition, we establish a preliminary lemma.

Lemma 15 Let $\left(P_{n}\right)_{n \in \mathbb{N}}$ and $P$ be probability measures on $S$. Suppose that for all $E \subseteq S$, $P_{n}(E)$ converges to $P(E)$. Fix $\alpha>0$ and let $V_{n}: S \rightarrow[-\alpha, \alpha]$ be a sequence of functions pointwise converging to $V: S \rightarrow[-\alpha, \alpha]$. Then, $\sum_{s^{\prime} \in S} V_{n}\left(s^{\prime}\right) P_{n}(s) \rightarrow \sum_{s^{\prime} \in S} V\left(s^{\prime}\right) P\left(s^{\prime}\right)$.

Proof: Observe that $\left|V_{n}\right|$ is bounded by $\alpha$ and that $\sum_{s^{\prime} \in S} \alpha P_{n}\left(s^{\prime}\right)=\alpha$ converges to $\sum_{s^{\prime} \in S} \alpha P\left(s^{\prime}\right)=\alpha$. The result follows from Theorem 18 in Royden (1968, Chapter 11).

Proof of Proposition 14. Let us begin proving (b). Consider any converging subsequence $\left(\bar{V}_{n_{k}}\right)_{k \in \mathbb{N}}$ to $\bar{V}_{0}$ (such a subsequence always exists). Fix $s \in S$. Consider any sequence $x_{k} \rightarrow x$ in X. Since $\bar{V}_{n_{k}}, \bar{V}_{0}$ are uniformly bounded by the same constant, we can apply Lemma 15 above to deduce that for any $x_{k} \rightarrow x, \sum_{s^{\prime} \in S} \bar{V}_{n_{k}}\left(s^{\prime}\right) \nu_{n_{k}}\left(s^{\prime} ; x_{k}, s\right) \rightarrow \sum_{s^{\prime} \in S} \bar{V}_{0}\left(s^{\prime}\right) \nu\left(s^{\prime} ; x, s\right)$. So, defining $\psi_{n}(x, s)=\sum_{s^{\prime} \in S} \bar{V}_{n}\left(s^{\prime}\right) \nu_{n}\left(s^{\prime} ; x, s\right)$ and $\psi_{0}(x, s)=\sum_{s^{\prime} \in S} \bar{V}_{0}\left(s^{\prime}\right) \nu\left(s^{\prime} ; x, s\right)$, we deduce that for all $s, \psi_{n_{k}}(\cdot, s)$ converges to $\psi_{0}(\cdot, s)$ uniformly on the compact set $X$.

Fix now $x \in X$. Condition 1. in Proposition 14 permits us to deduce that for all $x_{k} \rightarrow x$

$$
\limsup _{k \rightarrow \infty} \pi_{n_{k}}\left(x_{k}, s\right)+\delta \psi_{n_{k}}\left(x_{k}, s\right) \leq \pi(x, s)+\delta \psi(x, s)
$$

Additionally, there exists a sequence $y_{k}$ such that

$$
\liminf _{k \rightarrow \infty} \pi_{n_{k}}\left(y_{k}, s\right)+\delta \psi_{n_{k}}\left(y_{k}, s\right) \geq \pi(x, s)+\delta \psi(x, s)
$$

To prove this result, define $\varphi_{n_{k}}(x, s)=\pi_{n_{k}}(x, s)+\delta \sum_{s^{\prime} \in S} \bar{V}_{n_{k}}\left(s^{\prime}\right) \nu_{n_{k}}\left(s^{\prime} ; x, s\right)$ and $\varphi_{0}(x, s)=$ $\pi(x, s)+\delta \sum_{s^{\prime} \in S} \bar{V}_{0}\left(s^{\prime}\right) \nu\left(s^{\prime} ; x, s\right)$. Fix $\eta>0$. From Condition 2. in the Proposition, there exists $\hat{x}_{k} \rightarrow x$ in $X$ such that $\pi_{n_{k}}\left(\hat{x}_{k}, s\right) \geq \pi(x, s)-\frac{\eta}{3}$. We further know that the function $x \in$ $X \mapsto \delta \sum_{s^{\prime} \in S} \bar{V}_{n_{k}}\left(s^{\prime}\right) \nu_{n_{k}}\left(s^{\prime} ; x, s\right)$ converges continuously to $x \in X \mapsto \delta \sum_{s^{\prime} \in S} \bar{V}_{0}\left(s^{\prime}\right) \nu\left(s^{\prime} ; x, s\right)$. Consequently, for $k$ big enough $\delta \sum_{s^{\prime} \in S} \bar{V}_{0}\left(s^{\prime}\right) \nu\left(s^{\prime} ; x, s\right)-\delta \sum_{s^{\prime} \in S} \bar{V}_{n_{k}}\left(s^{\prime}\right) \nu\left(s^{\prime} ; \hat{x}_{k}, s\right) \leq \frac{\eta}{3}$. 
Therefore,

$$
\begin{aligned}
\max _{x \in X} \varphi_{n_{k}}(x, s) & \geq \varphi_{n_{k}}\left(\hat{x}_{k}, s\right) \\
& \geq \pi(x, s)-\frac{\eta}{3}+\delta \sum_{s^{\prime} \in S} \bar{V}_{n_{k}}\left(s^{\prime}\right) \nu\left(s^{\prime} ; \hat{x}_{k}, s\right) \\
& \geq \pi(x, s)-\frac{\eta}{3}+\delta \sum_{s^{\prime} \in S} \bar{V}_{0}\left(s^{\prime}\right) \nu\left(s^{\prime} ; x, s\right)-\frac{\eta}{3}
\end{aligned}
$$

Taking $y_{k}$ to be a $\eta / 3$-maximizer of the maximization problem above, we deduce that for all $k$ big enough $\varphi_{n_{k}}\left(y_{k}, s\right) \geq \varphi(x, s)-\eta$. Taking liminf, inequality (6.4) follows.

With these preliminary results, we are in position to prove (b). We will prove that for any subsequence $\bar{V}_{n_{k}} \rightarrow \bar{V}_{0}$, where $\bar{V}_{0}$ is some function, $T \bar{V}_{0}=\bar{V}_{0}$. The result then follows from the uniqueness property stated in Theorem 13 . Let $\bar{x}_{n_{k}} \in \bar{X}_{n_{k}}\left(s ; \bar{V}_{n_{k}}\right), s \in S$. Since $X$ is compact, we assume without loss of generality that $\bar{x}_{n_{k}} \rightarrow \bar{x}$ (eventually through a subsequence). Let $x \in \bar{X}_{0}\left(s ; \bar{V}_{0}\right)$ and consider $y_{k}$ as in 6.4 . Then

$$
\varphi(x, s) \leq \liminf _{k \rightarrow \infty} \varphi_{n_{k}}\left(y_{k}, s\right) \leq \liminf _{k \rightarrow \infty} \varphi_{n_{k}}\left(\bar{x}_{n_{k}}, s\right) \leq \limsup _{k \rightarrow \infty} \varphi_{n_{k}}\left(\bar{x}_{n_{k}}, s\right) \leq \varphi(\bar{x})
$$

The first inequality is by construction of the sequence $y_{k}$. The second inequality follows since $\bar{x}_{n_{k}} \in \bar{X}_{n_{k}}\left(s ; \bar{V}_{n_{k}}\right)$. The third inequality follows by definition. The fourth inequality holds by virtue of 6.3 .

It follows that $\bar{x} \in \bar{X}_{0}\left(s ; \bar{V}_{0}\right)$ and that the sequence of inequalities above are actually equalities. Therefore, $\bar{V}_{0}(s)=\lim _{k \rightarrow \infty} \bar{V}_{n_{k}}(s)=\lim _{k \rightarrow \infty} T_{n_{k}} \bar{V}_{n_{k}}(s)=\lim _{k \rightarrow \infty} \varphi_{n_{k}}\left(\bar{x}_{n_{k}}, s\right)=$ $\varphi(\bar{x}, s)=T \bar{V}_{0}(s)$, proving the first part of the proposition. Finally, to see (a), just apply the argument above to $V_{n_{j}} \rightarrow V$. Finally, (c) follows from (a) and (b).

\section{References}

Aleskerov, F., H. Ersel, and D. Piontkovski (2011): Linear Algebra for Economists. Springer.

Aliprantis, C., And K. Border (2006): Infinite Dimensional Analysis: A Hitchhiker's Guide. Springer Verlag. 
Amir, R. (1996): "Continuous Stochastic Games of Capital Accumulation with Convex Transitions," Games and Economic Behavior, 15(2), 111-131.

Bajari, P., C. Benkard, and J. Levin (2007): "Estimating Dynamic Models of Imperfect Competition," Econometrica, 75(5), 1331-1370.

Bernheim, D., And D. RAY (1989): "Markov Perfect Equilibria in Altruistic Growth Economies with Production Uncertainty," Journal of Economic Theory, 47(1), 195-202.

Besanko, D., U. Doraszelski, Y. Kryukov, and M. SAtTerthwaite (2010): "Learning-by-doing, organizational forgetting, and industry dynamics," Econometrica, $78(2), 453-508$.

Curtat, L. (1996): "Markov Equilibria of Stochastic Games with Complementarities," Games and Economic Behavior, 17(2), 177-199.

Dasgupta, P., And E. Maskin (1986): "The Existence of Equilibrium in Discontinuous Economic Games, I: Theory," The Review of Economic Studies, 53(1), 1-26.

Doraszelski, U., AND J. Escobar (2010): "A Theory of Regular Markov Perfect Equilibria in Dynamic Stochastic Games: Genericity, Stability, and Purification," Theoretical Economics, 5(3), 369-402.

Doraszelski, U., And M. SAtTerthwaite (2010): "Computable Markov Perfect Industry Dynamics," The RAND Journal of Economics, 41(2), 215-243.

Duffie, D., J. Geanakoplos, A. Mas-Colell, and A. Mclennan (1994): "Stationary Markov Equilibria," Econometrica, 62(4), 745-781.

Dutta, P., And R. Sundaram (1992): "Markovian Equilibrium in a Class of Stochastic Games: Existence Theorems for Discounted and Undiscounted Models," Economic Theory, 2(2), 197-214.

Ericson, R., And A. PAkes (1995): "Markov-Perfect Industry Dynamics: A Framework for Empirical Work," Review of Economic Studies, 62(1), 53-82.

Federgruen, A. (1978): "On N-Person Stochastic Games with Denumerable State Space," Advances in Applied Probability, 10(2), 452-471.

GlicksBerG, I. (1952): "A Further Generalization of the Kakutani Fixed Point Theorem, with Application to Nash Equilibrium Points," Proceedings of the American Mathematical Society, 3(1), 170-174. 
Horst, U. (2005): "Stationary Equilibria in Discounted Stochastic Games with Weakly Interacting Players," Games and Economic Behavior, 51(1), 83-108.

Mas-Colell, A., M. Whinston, and J. Green (1995): Microeconomic Theory. Oxford University Press, Oxford.

Maskin, E., And J. Tirole (1988): "A Theory of Dynamic Oligopoly, II: Price Competition, Kinked Demand Curves, and Edgeworth Cycles," Econometrica, 56(3), 571-599.

(2001): "Markov Perfect Equilibrium, I: Observable Actions," Journal of Economic Theory, 100(2), 191-219.

Mertens, J., and T. Parthasarathy (1987): "Equilibria for Discounted Stochastic Games," Working paper, CORE Universite Catholique Louvain, Belgium.

NowaK, A. (2007): "On Stochastic Games in Economics," Mathematical Methods of Operations Research, 6(3), 513-530.

Nowak, A., And T. Raghavan (1992): "Existence of Stationary Correlated Equilibria with Symmetric Information for Discounted Stochastic Games," Mathematics of Operations Research, 17(3), 519-526.

Rockafellar, R., And J. Roger (2004): Variational Analysis. Springer.

Royden, H. (1968): Real Analysis. Macmillan, New York.

Shapley, L. (1953): "Stochastic Games," Proceedings of the National Academy of Sciences, 39(10), 1095-1100.

Stokey, N., And E. Lucas, R. With Prescott (1989): Recursive Methods in Economic Dynamics. Harvard University Press, Cambridge.

Whitт, W. (1980): "Representation and Approximation of Noncooperative Sequential Games," SIAM Journal on Control and Optimization, 18(1), 33-48. 